\title{
Association between autism spectrum disorder and inflammatory bowel disease: A systematic review and meta-analysis
}

\author{
Jong Yeob Kim ${ }^{1}$ (C) | Min Je Choi ${ }^{1}$ | Sungji Ha ${ }^{2}$ | Jimin Hwang ${ }^{3} \quad$ Ai Koyanagi ${ }^{4,5}$ | \\ Elena Dragioti $^{6}$ | Joaquim Radua ${ }^{7,8,9}$ | Lee Smith ${ }^{10}$ | Louis Jacob ${ }^{4,11}$ | \\ Gonzalo Salazar de Pablo ${ }^{12,13,14}$ | Seung Won Lee ${ }^{15}$ | Dong Keon Yon ${ }^{16}$ \\ Trevor Thompson $^{17}$ | Samuele Cortese ${ }^{18,19,20,21}$ | Gianluca Lollo ${ }^{22}$ | Chih-Sung Liang ${ }^{23,24}$ | \\ Che-Sheng Chu ${ }^{25,26,27,28}$ | Paolo Fusar-Poli ${ }^{12,29,30,31}$ | Keun-Ah Cheon ${ }^{2}$ ( ) \\ Jae Il Shin $^{32}$ (1) | Marco Solmi ${ }^{12,18,33,34,35}$ \\ ${ }^{1}$ Yonsei University College of Medicine, Seoul, South Korea \\ ${ }^{2}$ Department of Child and Adolescent Psychiatry, Department of Psychiatry, Institute of Behavioral Science in Medicine, Yonsei University College of Medicine, Seoul, \\ South Korea \\ ${ }^{3}$ Department of Epidemiology, Johns Hopkins Bloomberg School of Public Health, Baltimore, Maryland, USA \\ ${ }^{4}$ Research and Development Unit, Parc Sanitari Sant Joan de Déu/CIBERSAM, Universitat de Barcelona, Fundació Sant Joan de Déu, Sant Boi de Llobregat, \\ Barcelona, Spain \\ ${ }^{5}$ ICREA, Barcelona, Spain \\ ${ }^{6}$ Pain and Rehabilitation Centre, and Department of Health, Medicine and Caring Sciences, Linköping University, Linköping, Sweden \\ ${ }^{7}$ Mental Health Research Networking Center (CIBERSAM), Institut d'Investigacions Biomèdiques August Pi i Sunyer (IDIBAPS), Barcelona, Spain \\ ${ }^{8}$ Department of Psychosis Studies, Institute of Psychiatry, Psychology and Neuroscience, King's College London, London, UK \\ ${ }^{9}$ Department of Clinical Neuroscience, Centre for Psychiatric Research, Karolinska Institutet, Stockholm, Sweden \\ ${ }^{10}$ Centre for Health, Performance, and Wellbeing, Anglia Ruskin University, Cambridge, UK \\ ${ }^{11}$ Faculty of Medicine, University of Versailles Saint-Quentin-en-Yvelines, Montigny-le-Bretonneux, France \\ ${ }^{12}$ Early Psychosis: Interventions and Clinical-detection (EPIC) Lab, Department of Psychosis Studies, Institute of Psychiatry, Psychology \& Neuroscience, King's College \\ London, London, UK \\ ${ }^{13}$ Child and Adolescent Mental Health Services, South London \& Maudsley NHS Trust, London, UK \\ ${ }^{14}$ Department of Child and Adolescent Psychiatry, Institute of Psychiatry, Psychology \& Neuroscience, King's College London, London, UK \\ ${ }^{15}$ Department of Data Science, Sejong University College of Software Convergence, Seoul, South Korea \\ ${ }^{16}$ Department of Pediatrics, Seoul National University College of Medicine, Seoul, South Korea \\ ${ }^{17}$ Centre for Chronic Illness and Ageing, University of Greenwich, London, UK \\ ${ }^{18}$ Centre for Innovation in Mental Health, School of Psychology, Faculty of Environmental and Life sciences \& Clinical and Experimental Sciences (CNS and \\ Psychiatry), Faculty of Medicine, University of Southampton, Southampton, UK \\ ${ }^{19}$ Solent NHS Trust, Southampton, UK \\ ${ }^{20}$ Division of Psychiatry and Applied Psychology, School of Medicine, University of Nottingham, Nottingham, UK \\ ${ }^{21}$ Hassenfeld Children's Hospital at NYU Langone, New York University Child Study Center, New York, New York, USA \\ ${ }^{22}$ Department of Gastroenterology, Ospedale Regionale di Bellinzona e Valli (Ente Ospedaliero Cantonale: EOC), Bellinzona, Switzerland \\ ${ }^{23}$ Department of Psychiatry, Beitou Branch, Tri-Service General Hospital, School of Medicine, National Defense Medical Center, Taipei, Taiwan \\ ${ }^{24}$ Graduate Institute of Medical Sciences, National Defense Medical Center, Taipei, Taiwan \\ ${ }^{25}$ Department of Psychiatry, Kaohsiung Veterans General Hospital, Kaohsiung, Taiwan \\ ${ }^{26}$ Center for Geriatric and Gerontology, Kaohsiung Veterans General Hospital, Kaohsiung, Taiwan \\ ${ }^{27}$ Society of Psychophysiology, Non-invasive Neuromodulation Consortium for Mental Disorders, Taipei, Taiwan \\ ${ }^{28}$ Graduate Institute of Medicine, College of Medicine, Kaohsiung Medical University, Kaohsiung, Taiwan
}

Jong Yeob Kim, Min Je Choi, and Sungji Ha contributed equally to this study as co-first authors.

Jae Il Shin and Keun-Ah Cheon are joint co-corresponding authors.

(C) 2021 International Society for Autism Research and Wiley Periodicals LLC. 


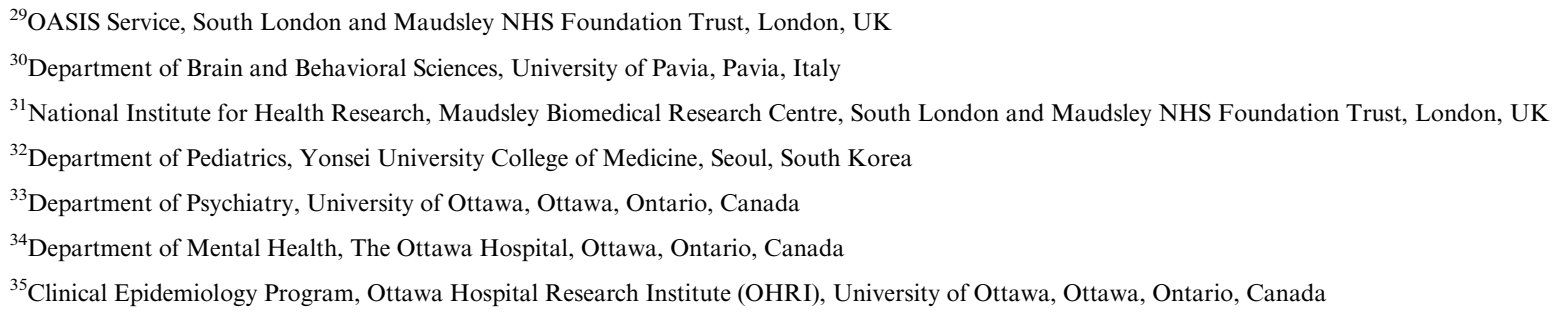

\section{Correspondence}

Jae Il Shin, Department of Pediatrics, Yonsei University College of Medicine, 50 Yonsei-ro, Seodaemun-gu, C.P.O. Box 8044, Seoul 03722 , South Korea

Email: shinji@yuhs.ac

Keun-Ah Cheon, Department of Child and Adolescent Psychiatry, Department of Psychiatry, Institute of Behavioral Science in Medicine, Yonsei University College of Medicine, 50 Yonsei-ro, Seodaemun-gu, C.P.O. Box 525, Seoul 03722, South Korea. Email: kacheon@yuhs.ac

Funding information

Korea government (MSIT), Grant/Award Number: 2021R1A2C2010913; National Research Foundation of Korea (NRF)

\section{INTRODUCTION}

Autism spectrum disorder (ASD) is a heterogeneous and hereditary neurodevelopmental disorder, characterized by impairments in social communication and interaction, repetitive behaviors, and restricted interests (American Psychiatric Association, 2013). According to the 2010 Global Burden of Disease study, 52 million people were estimated to have autism globally, equivalent to a prevalence of 7.6 per 1000 individuals (Baxter et al., 2015). In the United States, ASD prevalence was 18.5 per 1000 (one in 54) children aged 8 years in 2016, a nearly $175 \%$ increase than baseline estimates established in 2000 (Shaw et al., 2020). In addition to psychiatric comorbidities, many children with ASD are diagnosed with co-occurring medical conditions, including gastrointestinal (GI) disorders (Buie et al., 2010; Mannion \& Leader, 2014). Among the GI diseases, inflammatory bowel diseases (IBD), including Crohn's disease (CD) and ulcerative colitis (UC), are characterized by a 
chronic inflammatory condition in the GI tract, featuring symptoms including abdominal pain, diarrhea, bleeding, weight loss, with severe complications including bowel stenosis or perforation with need for surgery.

A number of pathophysiological hypotheses, focusing on putative gut-brain connections, have been put forward to explain the possible links between ASD and IBD, including a dysfunction in the intestinal tight junction in ASD, which leads to a change in the gut's permeability (d'Eufemia et al., 1996) and distinctive gut microbiomes in children with ASD compared to neurotypical children (Adams et al., 2011; Gondalia et al., 2012). The possibility of a common genetic background between ASD and IBD has also been proposed (Somekh et al., 2016). Several observational studies have reported a statistically significant association between ASD and IBD (Alexeeff et al., 2017; Butwicka et al., 2019; Doshi-Velez et al., 2015; Kohane et al., 2012; Lee et al., 2018). However, to the best of our knowledge, no systematic review has yet been performed to synthesize the existing evidence to provide the most robust and precise evaluation of the association. We filled this gap by conducting the first systematic review and metaanalysis to measure the association between ASD and subsequent IBD, and the association between the two conditions regardless of their temporal sequence.

\section{METHODS}

This systemic review and meta-analysis followed the recommendations of the Preferred Reporting Items for Systematic Reviews and Meta-Analyses statement 2020 (Appendix S1, page 3-5) (Page et al., 2021). The protocol of this systematic review was registered in PROSPERO (ID: CRD42021236263).

\section{Literature search strategy and eligibility criteria}

We systematically searched PubMed/ MEDLINE, Embase, and PsycInfo from inception to January 25,2021 , to identify eligible articles, using search keywords including "autism", "Asperger", "inflammatory bowel disease", "ulcerative colitis", and "Crohn" (see Appendix S1, page 6 for full search strategy). There was no language restriction. To identify eligible articles, two investigators (J. Y. K. and M. J. C.) independently screened titles, abstracts, and full-texts (Figure 1). We additionally manually searched the reference list of relevant publications for further potential eligible articles. Any disagreement was solved by reaching a consensus between three authors (J. Y. K., M. J. C., and J. I. S.).

We included primary observational studies (cohort studies, case-control studies, and cross-sectional studies) that reported estimates of the association between ASD and IBD or that provided data necessary to calculate them. We excluded reviews and conference abstracts. We excluded studies with fewer than 10 participants in either the ASD or IBD group. We used the Newcastle-Ottawa Scale (NOS) for quality assessment of the eligible studies (Wells et al., 2021) and studies with a NOS score of 7 or higher and between 4 and 6 were considered high and moderate quality, respectively.

\section{Data extraction}

We extracted the following data from the eligible studies: first author's last name, publication year, data sources, ASD diagnostic method (medical record, structured interview, self-report, parental/teacher report, or clinical diagnosis), IBD diagnostic method (medical report, endoscopic diagnosis, biopsy, or self-report), type of IBD (UC, CD, or unclassified IBD), number of ASD cases, IBD cases, and total participants, mean age and proportion of females of the population, maximally adjusted association estimate and types of variables adjusted for, unadjusted estimates from cross-sectional studies and timepoint prevalence ratio estimates from longitudinal studies, and effect metrics such as odds ratio (OR), risk ratio, and hazard ratio (HR) with $95 \% \mathrm{CI}$, and study design. If necessary, we calculated effect estimates from data presented in text, tables, or figures in the eligible studies. In a longitudinal study by Doshi-Velez and colleagues (Doshi-Velez et al., 2015), Kaplan-Meier curves of IBD incidence comparing the ASD group and the non-ASD group was presented, but their HRs were not reported, so we extracted data from the Kaplan-Meier curves and estimated the HRs using validated methods (Guyot et al., 2012; Saluja et al., 2019).

\section{Data analysis}

We performed meta-analyses under the random-effects model, which allows the true effect size to differ among cohorts (DerSimonian \& Laird, 1986). For the primary outcome, we aimed to evaluate if patients diagnosed with ASD were more likely to later develop IBD than patients without ASD. We thus assumed a temporal order of ASD diagnosis first and IBD diagnosis second, given the significant difference in the mean age of onset and lack of overlap between the age of onset ranges of the two conditions (Johnston \& Logan, 2008; van't Hof et al., 2021). We combined maximally adjusted effect estimates of the incidence of IBD between the ASD and the non-ASD groups from longitudinal studies and maximally adjusted effects estimates of the association from cross-sectional studies. For the secondary outcome, we tested the association between the two conditions regardless of their order of diagnosis, by combining the unadjusted estimates from cross-sectional studies and timepoint prevalence ratio estimates from longitudinal studies. Heterogeneity among the included studies was estimated by $\mathrm{I}^{2}, \tau^{2}$, and Cochran's Q-test, and $\mathrm{I}^{2}$ above 50\% was considered high 


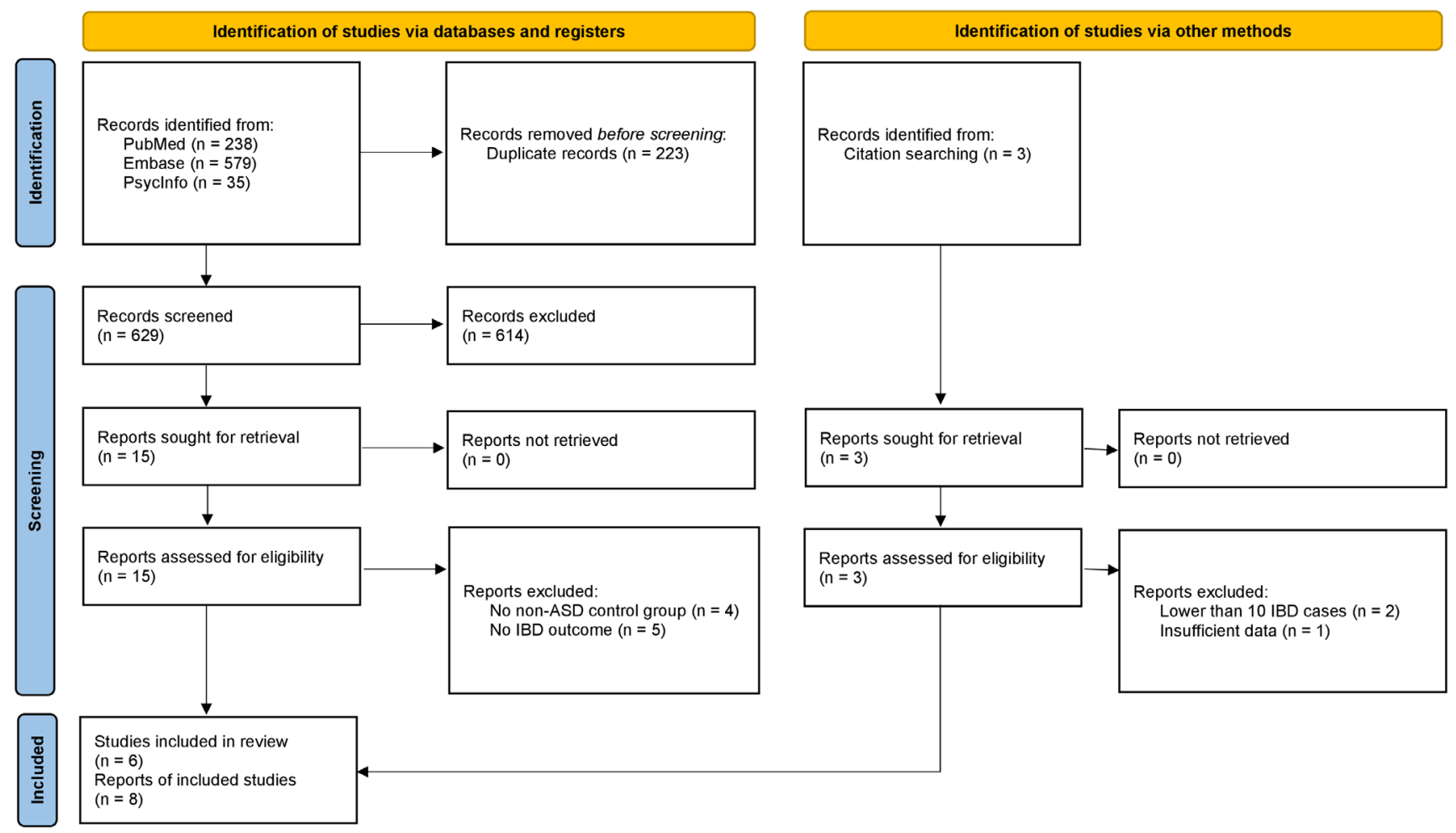

F I G U R E 1 Flow chart of literature screening and study selection

true heterogeneity (Higgins \& Thompson, 2002). We assessed the presence of publication bias using the pvalue of regression asymmetry test proposed by Egger and colleagues (Egger et al., 1997) and by visual inspection of the funnel plot. We additionally performed analyses by types of IBD, namely UC and CD. We performed subset analyses of longitudinal data, crosssectional data, high-quality studies, moderate quality studies, effect estimates adjusted for sex, and effect estimates not adjusted for sex. We performed sensitivity analysis by excluding one study at a time. We performed meta-regression analyses including mean age of the total population, proportion of females in total population, and NOS score. All statistical tests were two-sided. Statistical analysis was performed using R (R Foundation for Statistical Computing, version 4.0.5) with "the metafor package".

\section{RESULTS}

A total of 852 references were identified by the initial search (Figure 1). In the screening process, 12 articles were excluded after text screening (list of the excluded studies, with reason of exclusion, can be found in Appendix S1, page 7), and six eligible articles corresponding to eight datasets were finally included in our analysis (Table 1) (Alexeeff et al., 2017; Butwicka et al., 2019; Chen et al., 2013; Doshi-Velez et al., 2015; Kohane et al., 2012;
Lee et al., 2018). The total dataset for the meta-analysis included 129,678 ASD cases, 42,910 IBD cases, and $11,077,243$ total participants. Six of the datasets were US cohorts (Alexeeff et al., 2017; Doshi-Velez et al., 2015; Kohane et al., 2012; Lee et al., 2018), while one was a Swedish cohort (Butwicka et al., 2019) and one was a Taiwanese cohort (Chen et al., 2013). Three datasets were case-control studies comparing the ASD and the nonASD groups (Alexeeff et al., 2017; Chen et al., 2013; Lee et al., 2018), one dataset was a cross-sectional study (Kohane et al., 2012), three datasets were retrospective longitudinal cohort studies which followed ASD and non-ASD populations (Doshi-Velez et al., 2015), and one dataset (Butwicka et al., 2019) was a longitudinal study which followed childhood-onset IBD patients and controls. All but one (Butwicka et al., 2019) datasets were included in the primary analysis, and all datasets were included in the secondary analysis. While all studies ascertained ASD cases with International Classification of Diseases (ICD)-9/10 medical codes, none clarified whether more precise diagnosis methods like Autism Diagnostic Observation Schedule (ADOS) were used (Table 1). ASD diagnosis qualification was clarified in two datasets: Alexeeff and colleagues defined ASD cases as either diagnosis by a pediatric ASD specialist or two or more ASD diagnoses from non-specialists (Alexeeff et al., 2017), and Chen and colleagues defined ASD cases as diagnosis by board-certificated psychiatrists (Chen et al., 2013). While all studies ascertained IBD cases with 


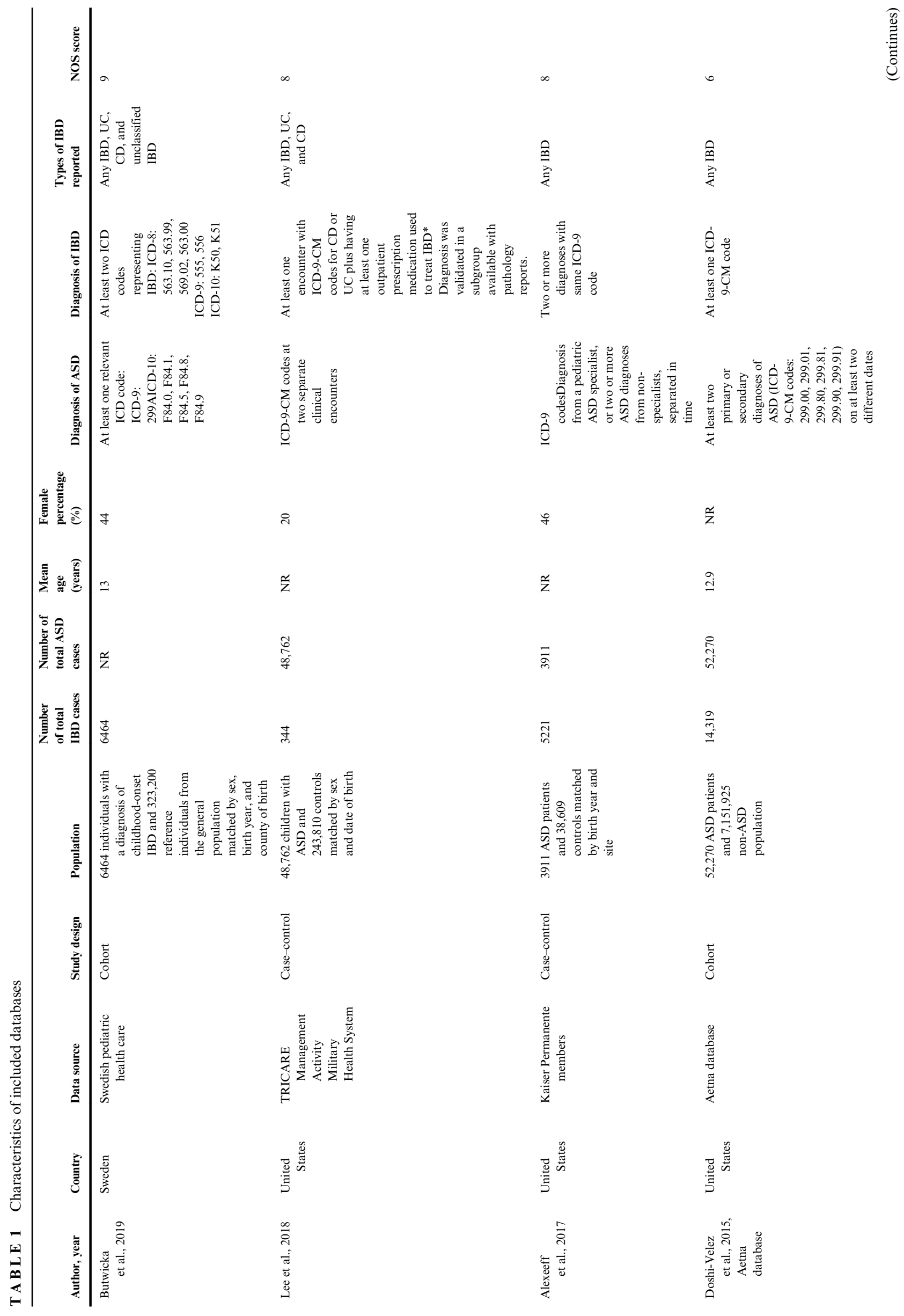




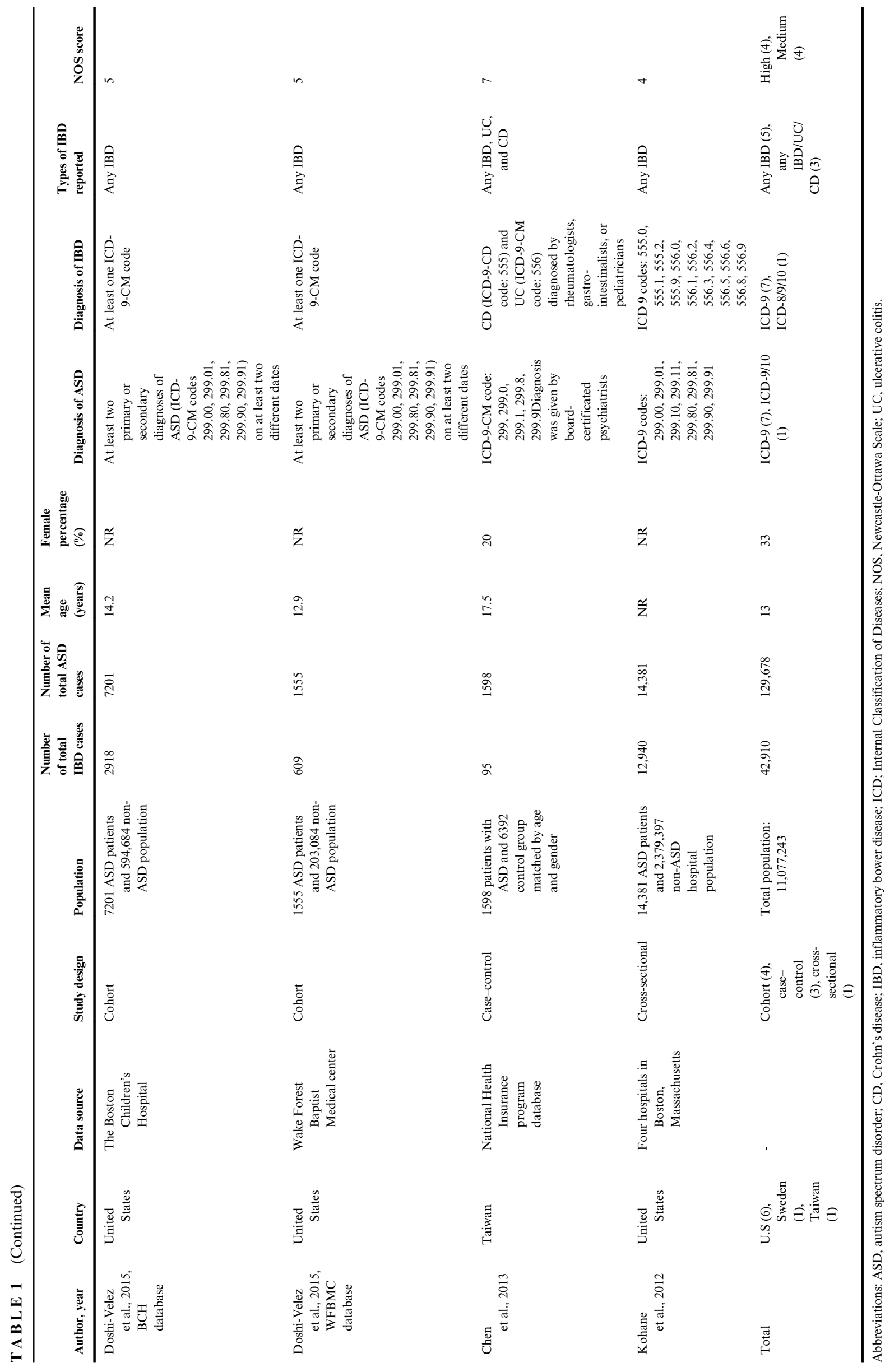


ICD-8/9/10 medical codes, none provided further details in method of diagnosis except for Lee and colleagues who confirmed their medical code-based IBD diagnosis using endoscopic pathology reports of a subgroup of IBD cases (Lee et al., 2018). IBD diagnosis qualification was clarified in only one dataset, where UC and CD were diagnosed by rheumatologists, gastro-intestinalists, or pediatricians (Chen et al., 2013). All studies adjusted for the age of participants as a potential confounder either by matching the control population to the exposure population by age (Alexeeff et al., 2017; Butwicka et al., 2019; Chen et al., 2013; Lee et al., 2018), adjusting for age covariate in statistical analysis (Doshi-Velez et al., 2015), or by applying age subgroups (Kohane et al., 2012). Four of the datasets adjusted for sex as a covariate (Alexeeff et al., 2017; Butwicka et al., 2019; Chen et al., 2013; Lee et al., 2018). NOS scores ranged from 4 to 9, and four datasets (Alexeeff et al., 2017; Butwicka et al., 2019; Chen et al., 2013; Lee et al., 2018) were rated high quality, and the rest (Doshi-Velez et al., 2015; Kohane et al., 2012) were rated moderate quality. Full details on quality assessment of each included study are provided in Appendix S1 (page 28-35).

The primary analysis, which evaluates if patients diagnosed with ASD were more likely to develop IBD than patients without ASD, showed that being diagnosed with ASD was positively associated with an increased risk of $\mathrm{IBD}(\mathrm{OR}=1.66,95 \% \mathrm{CI}=1.25$ to 2.21 , $p<0.001$ ) (Figure 2, Table 1). The association remained statistically significant when the type of IBD was restricted to $\mathrm{UC}(\mathrm{OR}=1.91,95 \% \mathrm{CI}=1.41$ to 2.6 , $p<0.001)$ or $\mathrm{CD}(\mathrm{OR}=1.47,95 \% \mathrm{CI}=1.15$ to 1.88 , $p=0.002)$. Secondary analysis, which tests the association between the two conditions regardless of their order of diagnosis, showed that ASD and IBD were positively associated, with slightly decreased effect sizes compared to primary analysis (any IBD, $\mathrm{OR}=1.57,95 \% \mathrm{CI}=1.28$ to $1.93, p<0.001$; $\mathrm{UC}, \mathrm{OR}=1.7,95 \% \mathrm{CI}=1.36$ to $2.12, p<0.001 ; \mathrm{CD}, \mathrm{OR}=1.37,95 \% \mathrm{CI}=1.12$ to 1.69 , $p=0.003$ ) (Figure 3, Table 1). Heterogeneity was high in both primary $\left(\mathrm{I}^{2}=93 \%, \tau^{2}=0.122\right)$ and secondary analysis $\left(\mathrm{I}^{2}=87 \%, \tau^{2}=0.066\right)$. However, the high $\mathrm{I}^{2}$ means that a large proportion $(93 \%$ and $87 \%$ in primary and secondary analysis, respectively) of the variability in effect sizes is due to true effect size variation across the studies, not that the effect sizes are particularly spread out (Borenstein et al., 2017), as can be observed in Figures 2 and 3 . The high $\mathrm{I}^{2}$ reflects little random error due to big sample size of an individual study, namely the Aetna dataset reported by Doshi-Velez and colleagues (Doshi-Velez et al., 2015) consisting of more than 7 million patients, which reported effect estimates large and precise compared to other datasets $(\mathrm{HR}=2.77,95 \%$ $\mathrm{CI}=2.44$ to 3.14 in the primary analysis; $\mathrm{OR}=2.40$, $95 \% \mathrm{CI}=2.11$ to 2.73 in the secondary analysis). Surely, when we excluded the Aetna dataset during our sensitivity analysis, $\mathrm{I}^{2}$ and decreased to $0 \%$ in both primary and secondary analyses while retaining statistical significance with similar summary effect sizes $(\mathrm{OR}=1.42,95 \%$ $\mathrm{CI}=1.31$ to $1.53, p<0.001$ in primary analysis; $\mathrm{OR}=1.41,95 \% \mathrm{CI}=1.31$ to $1.51, p<0.001$ in secondary analysis) (Table 2). Statistical significance was retained in all types of subset analyses (Table 2). Some

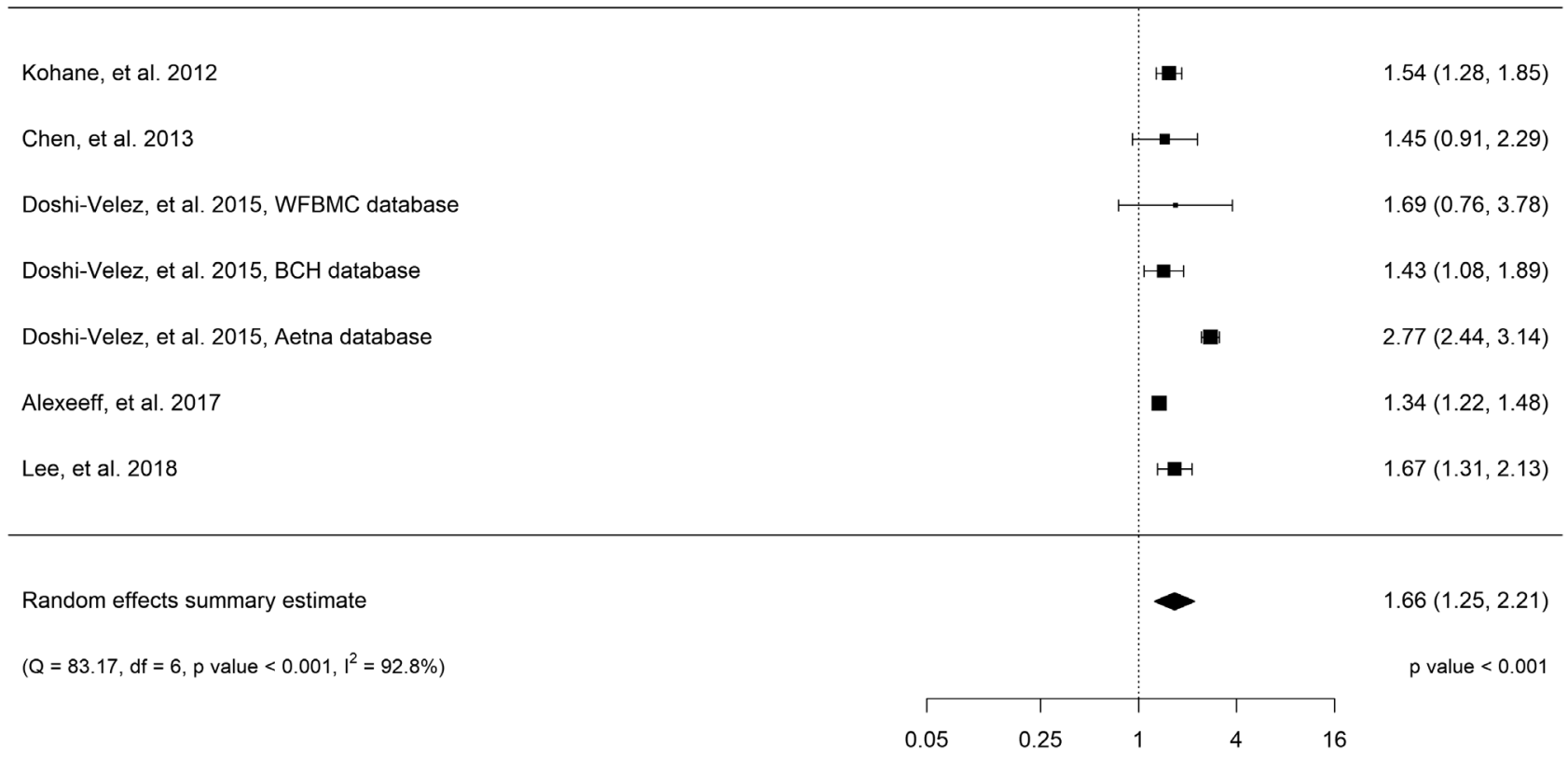

F I G U R E 2 Forest plot of risk of inflammatory bowel diseases in those with versus without autism spectrum disorders 


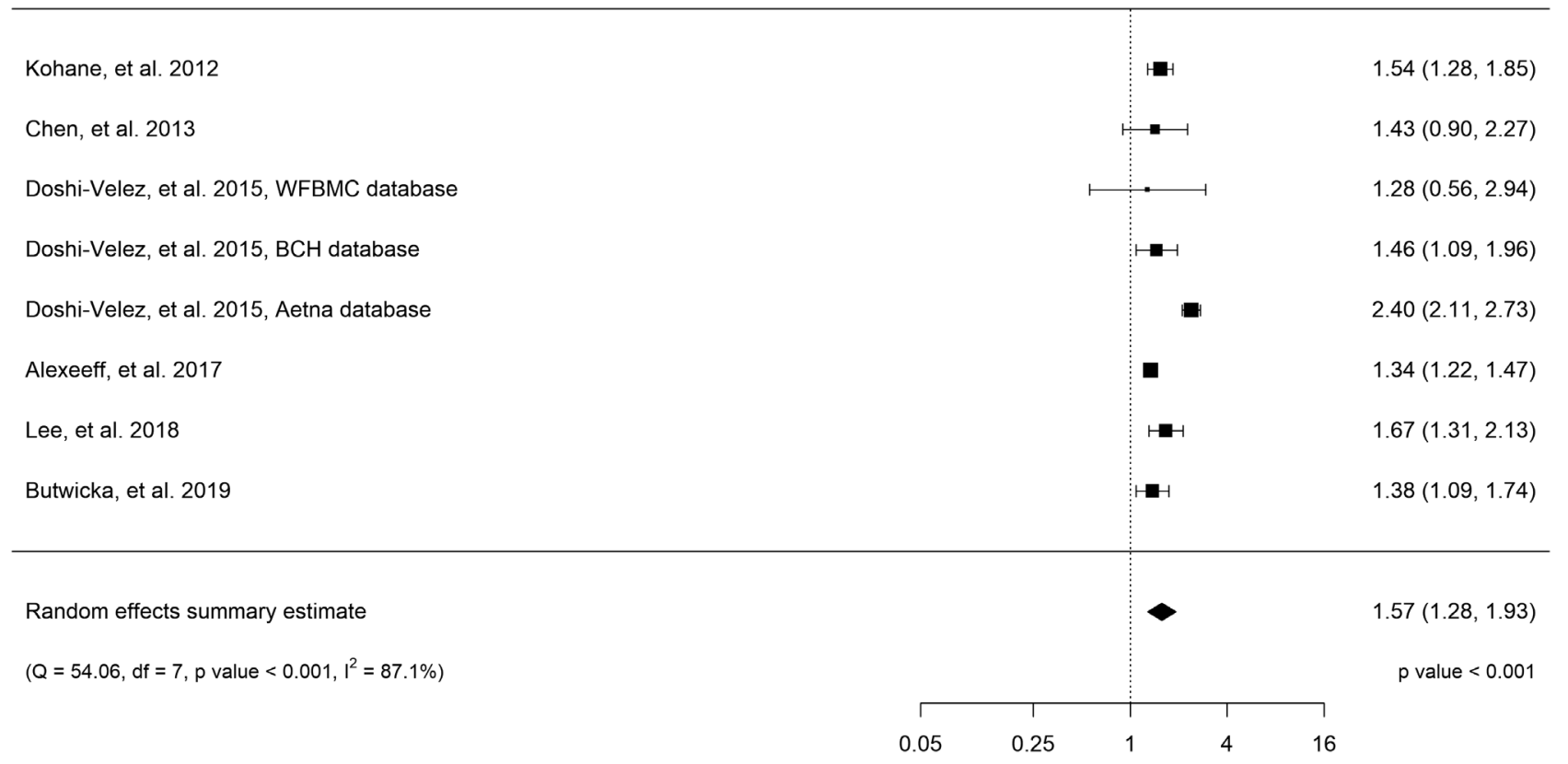

F I G U R E 3 Forest plot of association between autism spectrum disorders and inflammatory bowel diseases, any temporal sequence

subset analyses reported large heterogeneity $\left(\mathrm{I}^{2}>50 \%\right)$. However, all these subsets included the Aetna dataset reported by Doshi-Velez and colleagues (Doshi-Velez et al., 2015), and when we excluded the dataset from the analyses, $\mathrm{I}^{2}$ decreased to below $50 \%$ (Appendix S1, page 27). Forest plots of all analyses are provided in Appendix S1, pages 11-27. There was no evidence of publication bias (Appendix S1, page 9-10), and metaregression by mean age, female percentage, and NOS score did not show significant results (Appendix S1, page 8). However, Egger's test and meta-regression analysis should be interpreted with caution because 10 or more study estimates are typically required for these tests to be meaningful (Borenstein et al., 2009).

\section{DISCUSSION}

Whilst many studies on the relationship between ASD and IBD have been reported, to our knowledge, this is the first systematic review and meta-analysis to investigate the association between ASD and IBD. In our primary analysis, which assumed temporal order of ASD diagnosis to IBD diagnosis, we found that individuals diagnosed with ASD had a 1.66-fold increased risk of being diagnosed with IBD later in life, and in our secondary analysis, which estimated the association regardless of the order of diagnoses, ASD diagnosis and IBD diagnosis was positively associated. The associations were also statistically significant when restricted to UC or CD, with the magnitude of the association being larger in UC than in CD. Our findings sum up to the paramount literature mapping the association between mental disorders and physical comorbidities, informing clinical practice (Cortese et al., 2020).

Our primary analysis suggests that screening for IBD in children with ASD is warranted. Difficulties in IBD diagnosis have been reported due to the long prodromal period of abdominal symptoms which are nonspecific and could be mistakenly labeled as functional or benign (Burgmann et al., 2006). Diagnostic overshadowing of mental disorders over medical conditions is a well-known indirect burden affecting people with other mental conditions as well, that leads to poor physical healthcare, beyond any age or diagnostic boundaries (Firth et al., 2019). For instance, individuals with schizophrenia or mood disorders receive less frequent screening or treatment for cancer or cardiovascular disease (Solmi et al., 2020). For this reason, in the case of ASD and IBD, there are significant time delays between first IBD symptoms and IBD diagnosis, consisting of the patientdependent period (from first symptoms to physician visit) and physician-dependent period (from physician visit to IBD diagnosis) (Vavricka et al., 2012), which can lead to poor prognosis (Nguyen et al., 2017; Zaharie et al., 2016). In particular, diagnostic delay of CD was significantly correlated with increased complications including intestinal strictures, bowel stenosis, and IBDrelated intestinal surgeries (Nguyen et al., 2017; Zaharie et al., 2016). These reports highlight the clinical need to identify patients at high risk of IBD in whom more rigorous screening for IBD may be beneficial. Additionally, more than $20 \%$ of IBD patients develop IBD in childhood, and IBD can affect children's physiological and 


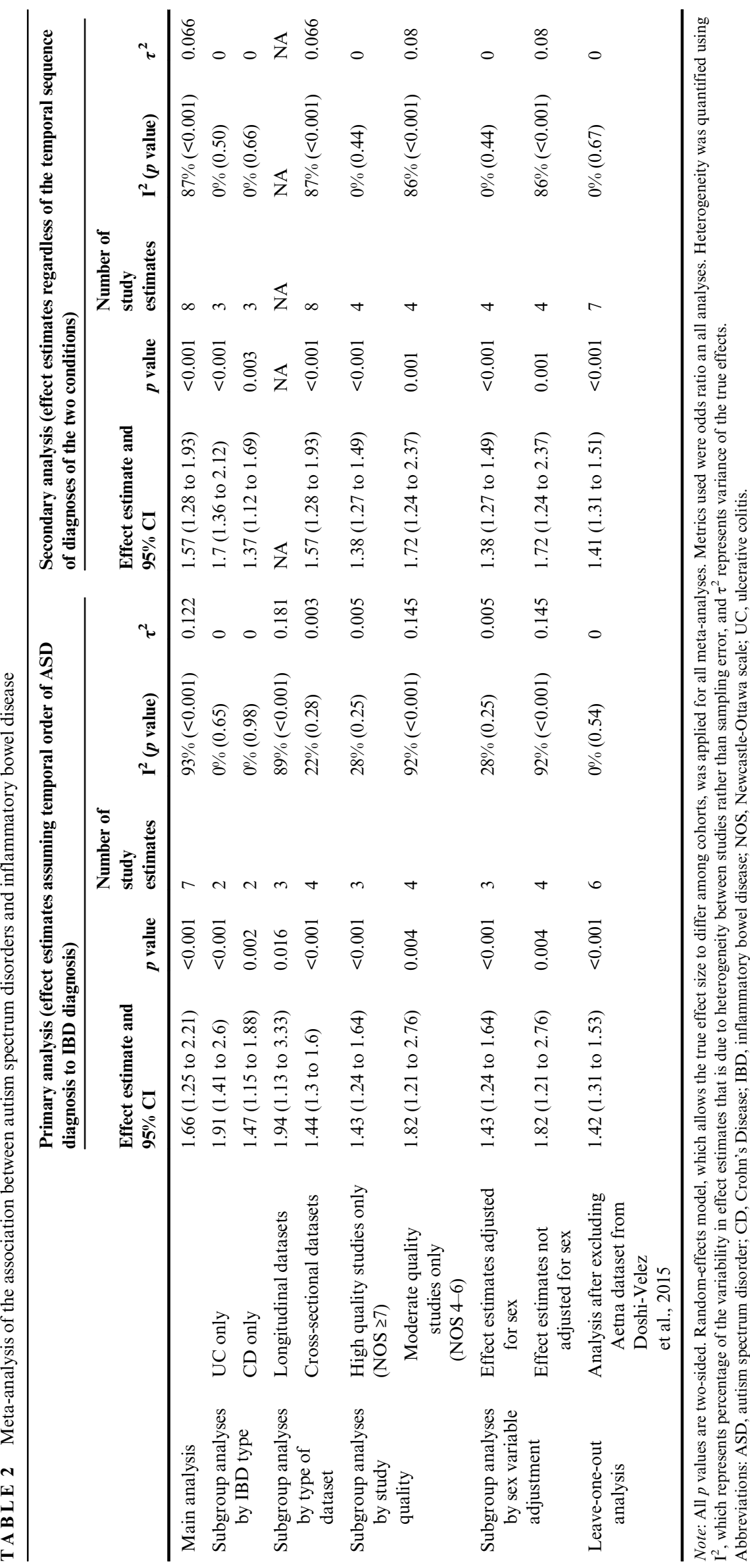


emotional development (Abramson et al., 2010; Mamula et al., 2003). According to the American Academy of Pediatrics, there are many co-occurring medical comorbidities in ASD, including GI disorders like IBD, and these comorbidities have a significant effect on the quality of life of children with ASD and their families (Hyman et al., 2020). In particular, children with ASD may have more severe phenotypes of IBD, as Lee and colleagues suggested through the fact that the rate of prescription of second-tier biologics was higher in the ASD group than in the non-ASD group among IBD patients (Lee et al., 2018). As greater severity of IBD symptoms is generally associated with lower quality of life patients with IBD (Habibi et al., 2017), we can expect that patients with both ASD and IBD will have even lower quality of life than patients with only IBD.

Despite this importance of early diagnosis of IBD, currently there is no guideline highlighting the need for early screening and treatment of IBD in patients with ASD (Hyman et al., 2020), nor there is universally accepted method for screening IBD in high-risk groups. Screening for typical symptoms of IBD such as abdominal pain, diarrhea, rectal bleeding, and weight loss may serve as a simple but useful screening method, however with somewhat low sensitivity ranging from $48 \%$ to $82 \%$ according to a meta-analysis (Holtman, Lisman-van Leeuwen, Reitsma, \& Berger, 2016). Fecal calprotectin has been suggested as a non-invasive and highly sensitive biomarker, with meta-analyses reporting pooled sensitivity ranging from $85 \%$ to $99 \%$ (Henderson et al., 2014; Holtman et al., 2016; Rokkas et al., 2018), but consensus should be reached on the optimal cutoff value of calprotectin, where studies have suggested cutoffs of $>200,100$, and $50 \mathrm{ug} / \mathrm{g}$. (Degraeuwe et al., 2015; Henderson et al., 2014; Kostakis et al., 2013). Out of serologic marker candidates, C-reactive protein and albumin had best performance, but had low sensitivity, respectively $63 \%$ and $48 \%$ according to a meta-analysis (Holtman et al., 2016), to be appropriate for screening method. While esophagogastroduodenoscopy and colonoscopy and consequent pathology may confirm diagnosis of IBD in suspected patients, these tests are often difficult for children (Henderson et al., 2014). Intestinal ultrasound to detect increased bowel wall thickening and other signs of IBD is an alternative non-invasive method of diagnosis with relatively high sensitivity and specificity (Dilillo et al., 2019; Nardo et al., 2020). Clinicians should acknowledge the high possibility of IBD in patients with ASD who report GI symptoms, and future guidelines for early screening of IBD with non-invasive tests and vigilant monitoring of GI symptoms in patients with ASD must be established, which can serve as important strategies to improve prognosis and quality of life for patients with ASD.

Our secondary analysis showed that ASD and IBD were positively associated with an OR of 1.57 (95\%
$\mathrm{CI}=1.28-1.93)$. This result provides further evidence for the possibility that ASD and IBD have a common etiological mechanism. Since the pathological mechanisms of disease for both ASD and IBD have not been clearly elucidated so far, it is difficult to establish a common mechanism between them, but several possible mechanisms have been suggested. First of all, there are commonalities at the genetic and molecular level. Somekh and colleagues found genes involving autophagy dysregulation whose loss of function mutations affect susceptibility to both ASD and IBD (Somekh et al., 2016). Walker and colleagues also found a significant overlapping GI mucosal molecular profiles between patients with ASD and IBD (Walker et al., 2013). It also has been suggested that the decrease in gene expression associated with disaccharidase expression and hexose transporters found in children with ASD possibly induce GI symptoms observed in ASD children (Williams et al., 2011). Second, immune dysfunction may be linked to both conditions. Various immune abnormalities including imbalanced cytokine response, differences in the number and distribution of immune cells and neuro-inflammation have been reported in ASD patients, which were also consistently found in patients with IBD (Hughes et al., 2018; Sanchez-Munoz et al., 2008). Third, alterations in gut microbiota may influence both the inflammatory state of the GI tract potentially associated with IBD (Khan et al., 2019). Truly, alterations in gut microbiota in both ASD group (Adams et al., 2011) and IBD group (Sartor \& Wu, 2017) have been reported in multiple previous studies. Although the cause-and-effect relationship between the gut microbiota and the two disorders is not yet clear, results from recent trials that fecal microbiota transplant therapy was associated with improvements in autism-related symptoms in individuals with ASD (Kang et al., 2019) and disease remission in individuals with UC (Moayyedi et al., 2015) support the hypothesis that alterations in gut microbiota is at least partially causative of the two disorders. Lastly, increasing evidence suggests role of microbiota-immune interactions as an important regulator of the gut-brain axis in central nervous system and related disorder. It has been reported that the gut microbiota not only modulates host's immune system, but also regulates the brain development and function and behavior (Fung, 2020; Fung et al., 2017). This is supported by findings from pre-clinical studies where transplantation of gut microbiota from individuals with ASD to germ-free mice induced autistic behaviors (Sharon et al., 2019), while treating maternal immune activation mouse model, known to display features of ASD, with human commensal bacterium resulted in altered microbial composition and improvements in behavioral and neuropathological features of ASD (Hsiao et al., 2013). Although more studies are needed to elucidate the common mechanisms of ASD and IBD, changes in the intestinal environment such as microbiome 
dysbiosis and the resulting imbalance in the immune system seem to be contributing significantly to the pathogenesis of these two diseases.

There are several potential limitations to this study. First, heterogeneity was high in both primary and secondary analyses $\left(\mathrm{I}^{2}>50 \%\right)$. However, the high $\mathrm{I}^{2}$ decreased to $0 \%$ when we excluded the Aetna dataset (Doshi-Velez et al., 2015) from the analysis, which reported a significantly larger effect size than the other datasets. Although we could not identify why the Aetna dataset reported an effect size which is significantly larger than other cohorts, we found that both primary and secondary analyses remained statistically significant even after the exclusion of the Aetna dataset, which is why we believe that the association between ASD and IBD is robust nevertheless. Second, Egger's test found no evidence of publication bias, but this must be interpreted with caution as the test typically requires at least 10 study estimates to have sufficient power, while our meta-analyses only included eight (Sterne et al., 2011). However, the funnel plot did not show asymmetry when visually inspected. Third, most included data in this study were from hospital or medical center settings, which can be biased toward patients of higher morbidity. Fourth, most eligible studies did not provide the method of diagnosis and qualification of diagnosis of ASD and IBD, leading to potential bias in our findings. Fifth, the studies did not provide potentially useful information, including effect estimates specific to sex or presence of intellectual disability, quantifications of severity of ASD and IBD, or change of severity with age. Future studies reporting such effect estimates or doseresponse association between severity of the two conditions will provide more robust evidence and add to clinical significance of the association. Also, a possible referral bias should be considered, whereby participants with a history of GI symptoms may be more likely to be recruited in research. Future studies will be needed to validate these findings in prospective studies of other populations with low referral bias such as community cohorts.

\section{CONCLUSION}

Despite some limitations, this systematic review and meta-analysis found a consistent and significant associations between ASD and IBD, providing comprehensive evidence for their potential association. Our findings may be useful evidence for early diagnosis and treatment of IBD among ASD patients with GI symptoms and may guide future molecular studies to elucidate the shared biological mechanisms between ASD and IBD.

\section{ACKNOWLEDGMENTS}

This research was supported by the Basic Science Research Program through the National Research Foundation of Korea (NRF) grant funded by the Korea government (MSIT) (2021R1A2C2010913).

\section{CONFLICT OF INTEREST}

Marco Solmi has been a consultant for/received honoraria from Angelini, Lundbeck. Samuele Cortese declares honoraria and reimbursement for travel and accommodation expenses for lectures from the following non-profit associations: Association for Child and Adolescent Central Health (ACAMH), Canadian ADHD Alliance Resource (CADDRA), British Association of Pharmacology (BAP), and from Healthcare Convention for educational activity on ADHD. Samuele Cortese's research is funded by the NIHR. Gonzalo Salazar de Pablo has been a consultant for/received honoraria from Janssen Cilaq and Angelini. Other authors have no conflict of interest to declare.

\section{AUTHORS CONTRIBUTION}

Jong Yeob Kim, Min Je Choi, and Jae Il Shin designed the study. Jong Yeob Kim, Min Je Choi, and Jae Il Shin did the literature search and screening, extracted, analyzed, and interpreted the data, and made the figures and tables. All authors drafted and critically revised the manuscript. All authors gave approval to the final version of the manuscript for publication. Marco Solmi provided overall supervision on the conduct of the study. All authors approved the final version of the manuscript for publication. The corresponding authors (Jae Il Shin and Keun-Ah Cheon) attest that all listed authors meet authorship criteria and that no others meeting the criteria have been omitted. Jong Yeob Kim, Min Je Choi, and Sungji Ha contributed equally to the manuscript as joint first authors.

\section{ORCID}

Jong Yeob Kim (1D https://orcid.org/0000-0003-4756-9440 Keun-Ah Cheon (1) https://orcid.org/0000-0001-8790-9708 Jae Il Shin (10 https://orcid.org/0000-0003-2326-1820

\section{REFERENCES}

Abramson, O., Durant, M., Mow, W., Finley, A., Kodali, P., Wong, A., \& Herrinton, L. J. (2010). Incidence, prevalence, and time trends of pediatric inflammatory bowel disease in northern California, 1996 to 2006. The Journal of Pediatrics, 157(2), 233-239.

Adams, J. B., Johansen, L. J., Powell, L. D., Quig, D., \& Rubin, R. A. (2011). Gastrointestinal flora and gastrointestinal status in children with autism-comparisons to typical children and correlation with autism severity. BMC Gastroenterology, 11(1), 1-13.

Alexeeff, S. E., Yau, V., Qian, Y., Davignon, M., Lynch, F., Crawford, P., \& Croen, L. A. (2017). Medical conditions in the first years of life associated with future diagnosis of ASD in children. Journal of Autism and Developmental Disorders, 47(7), 20672079.

American Psychiatric Association. (2013). Diagnostic and statistical manual of mental disorders, (DSM-5) (5th ed.). American Psychiatric Publishing.

Baxter, A. J., Brugha, T., Erskine, H. E., Scheurer, R. W., Vos, T., \& Scott, J. G. (2015). The epidemiology and global burden of autism spectrum disorders. Psychological Medicine, 45(3), 601.

Borenstein, M., Hedges, L. V., Higgins, J. P., \& Rothstein, H. R. (2009). Introduction to meta-analysis. John Wiley \& Sons. 
Borenstein, M., Higgins, J. P., Hedges, L. V., \& Rothstein, H. R. (2017). Basics of meta-analysis: $\mathrm{I}(2)$ is not an absolute measure of heterogeneity. Research Synthesis Methods, 8(1), 5-18.

Buie, T., Campbell, D. B., Fuchs, G. J., Furuta, G. T., Levy, J., VandeWater, J., \& Beaudet, A. L. (2010). Evaluation, diagnosis, and treatment of gastrointestinal disorders in individuals with ASDs: A consensus report. Pediatrics, 125(1), 1-18.

Burgmann, T., Clara, I., Graff, L., Walker, J., Lix, L., Rawsthorne, P., \& Bernstein, C. N. (2006). The Manitoba inflammatory bowel disease cohort study: Prolonged symptoms before diagnosis- How much is irritable bowel syndrome? Clinical Gastroenterology and Hepatology: The Official Clinical Practice Journal of the American Gastroenterological Association, 4(5), 614-620.

Butwicka, A., Olén, O., Larsson, H., Halfvarson, J., Almqvist, C., Lichtenstein, P., \& Ludvigsson, J. F. (2019). Association of childhood-onset inflammatory bowel disease with risk of psychiatric disorders and suicide attempt. JAMA Pediatrics, 173(10), 969-978.

Chen, M.-H., Su, T.-P., Chen, Y.-S., Hsu, J.-W., Huang, K.-L., Chang, W.-H., \& Bai, Y.-M. (2013). Comorbidity of allergic and autoimmune diseases in patients with autism spectrum disorder: A nationwide population-based study. Research in Autism Spectrum Disorders, 7(2), 205-212.

Cortese, S., Solmi, M., Arrondo, G., Cipriani, A., Fusar-Poli, P., Larsson, H., \& Correll, C. (2020). Association between mental disorders and somatic conditions: Protocol for an umbrella review. Evidence-Based Mental Health, 23(4), 135-139.

Degraeuwe, P. L., Beld, M. P., Ashorn, M., Canani, R. B., Day, A. S., Diamanti, A., \& Kessels, A. G. (2015). Faecal calprotectin in suspected paediatric inflammatory bowel disease. Journal of Pediatric Gastroenterology and Nutrition, 60(3), 339-346.

DerSimonian, R., \& Laird, N. (1986). Meta-analysis in clinical trials. Controlled Clinical Trials, 7(3), 177-188.

d'Eufemia, P., Celli, M., Finocchiaro, R., Pacifico, L., Viozzi, L., Zaccagnini, M., \& Giardini, O. (1996). Abnormal intestinal permeability in children with autism. Acta Paediatrica, 85(9), 10761079 .

Dilillo, D., Zuccotti, G. V., Galli, E., Meneghin, F., Dell'Era, A., Penagini, F., \& Maconi, G. (2019). Noninvasive testing in the management of children with suspected inflammatory bowel disease. Scandinavian Journal of Gastroenterology, 54(5), 586-591.

Doshi-Velez, F., Avillach, P., Palmer, N., Bousvaros, A., Ge, Y., Fox, K., \& Kohane, I. (2015). Prevalence of inflammatory bowel disease among patients with autism spectrum disorders. Inflammatory Bowel Diseases, 21(10), 2281-2288.

Egger, M., Davey Smith, G., Schneider, M., \& Minder, C. (1997). Bias in meta-analysis detected by a simple, graphical test. $B M J$, 315(7109), 629-634.

Firth, J., Siddiqi, N., Koyanagi, A., Siskind, D., Rosenbaum, S., Galletly, C., \& Stubbs, B. (2019). The lancet psychiatry commission: A blueprint for protecting physical health in people with mental illness. The Lancet, 6(8), 675-712.

Fung, T. C. (2020). The microbiota-immune axis as a central mediator of gut-brain communication. Neurobiology of Disease, 136, 104714.

Fung, T. C., Olson, C. A., \& Hsiao, E. Y. (2017). Interactions between the microbiota, immune and nervous systems in health and disease. Nature Neuroscience, 20(2), 145-155.

Gondalia, S. V., Palombo, E. A., Knowles, S. R., Cox, S. B., Meyer, D., \& Austin, D. W. (2012). Molecular characterisation of gastrointestinal microbiota of children with autism (with and without gastrointestinal dysfunction) and their neurotypical siblings. Autism Research: Official Journal of the International Society for Autism Research, 5(6), 419-427.

Guyot, P., Ades, A. E., Ouwens, M. J., \& Welton, N. J. (2012). Enhanced secondary analysis of survival data: Reconstructing the data from published Kaplan-Meier survival curves. BMC Medical Research Methodology, 12, 9.
Habibi, F., Habibi, M. E., Gharavinia, A., Mahdavi, S. B., Akbarpour, M. J., Baghaei, A., \& Emami, M. H. (2017). Quality of life in inflammatory bowel disease patients: A cross-sectional study. Journal of Research in Medical Sciences : The Official Journal of Isfahan University of Medical Sciences, 22, 104.

Henderson, P., Anderson, N. H., \& Wilson, D. C. (2014). The diagnostic accuracy of fecal calprotectin during the investigation of suspected pediatric inflammatory bowel disease: A systematic review and meta-analysis. The American Journal of Gastroenterology, 109(5), 637-645.

Higgins, J. P., \& Thompson, S. G. (2002). Quantifying heterogeneity in a meta-analysis. Statistics in Medicine, 21(11), 1539-1558.

Holtman, G. A., Lisman-van Leeuwen, Y., Reitsma, J. B., \& Berger, M. Y. (2016). Noninvasive tests for inflammatory bowel disease: A meta-analysis. Pediatrics, 137(1), e20152126.

Hsiao, E. Y., McBride, S. W., Hsien, S., Sharon, G., Hyde, E. R., McCue, T., \& Mazmanian, S. K. (2013). Microbiota modulate behavioral and physiological abnormalities associated with neurodevelopmental disorders. Cell, 155(7), 1451-1463.

Hughes, H. K., Ko, E. M., Rose, D., \& Ashwood, P. (2018). Immune dysfunction and autoimmunity as pathological mechanisms in autism spectrum disorders. Frontiers in Cellular Neuroscience, $12,405$.

Hyman, S. L., Levy, S. E., \& Myers, S. M. (2020). Identification, evaluation, and management of children with autism spectrum disorder. Pediatrics, 145(1), e20193447.

Johnston, R. D., \& Logan, R. F. (2008). What is the peak age for onset of IBD? Inflammatory Bowel Diseases, 14(2), 4-5.

Kang, D. W., Adams, J. B., Coleman, D. M., Pollard, E. L., Maldonado, J., McDonough-Means, S., \& Krajmalnik-Brown, R. (2019). Long-term benefit of microbiota transfer therapy on autism symptoms and gut microbiota. Scientific Reports, 9(1), 5821.

Khan, I., Ullah, N., Zha, L., Bai, Y., Khan, A., Zhao, T., \& Zhang, C. (2019). Alteration of gut microbiota in inflammatory bowel disease (IBD): Cause or consequence? IBD treatment targeting the gut microbiome. Pathogens, 8(3), 126.

Kohane, I. S., McMurry, A., Weber, G., MacFadden, D., Rappaport, L., Kunkel, L., \& Murphy, S. (2012). The comorbidity burden of children and young adults with autism spectrum disorders. PLoS One, 7(4), e33224.

Kostakis, I. D., Cholidou, K. G., Vaiopoulos, A. G., Vlachos, I. S., Perrea, D., \& Vaos, G. (2013). Fecal calprotectin in pediatric inflammatory bowel disease: A systematic review. Digestive Diseases and Sciences, 58(2), 309-319.

Lee, M., Krishnamurthy, J., Susi, A., Sullivan, C., Gorman, G. H., Hisle-Gorman, E., \& Nylund, C. M. (2018). Association of autism spectrum disorders and inflammatory bowel disease. Journal of Autism and Developmental Disorders, 48(5), 1523-1529.

Mamula, P., Markowitz, J. E., \& Baldassano, R. N. (2003). Inflammatory bowel disease in early childhood and adolescence: Special considerations. Gastroenterology Clinics of North America, 32(3), 967-995.

Mannion, A., \& Leader, G. (2014). Gastrointestinal symptoms in autism spectrum disorder: A literature review. Review Journal of Autism and Developmental Disorders, 1(1), 11-17.

Moayyedi, P., Surette, M. G., Kim, P. T., Libertucci, J., Wolfe, M., Onischi, C., \& Lee, C. H. (2015). Fecal microbiota transplantation induces remission in patients with active ulcerative colitis in a randomized controlled trial. Gastroenterology, 149(1), 102-109.

Nardo, G. D., Esposito, G., Ziparo, C., Micheli, F., Masoni, L., Villa, M. P., \& Corleto, V. D. (2020). Enteroscopy in children and adults with inflammatory bowel disease. World Journal of Gastroenterology, 26(39), 5944-5958.

Nguyen, V. Q., Jiang, D., Hoffman, S. N., Guntaka, S., Mays, J. L., Wang, A., \& Sorrentino, D. (2017). Impact of diagnostic delay and associated factors on clinical outcomes in a US inflammatory bowel disease cohort. Inflammatory Bowel Diseases, 23(10), 1825-1831. 
Page, M. J., McKenzie, J. E., Bossuyt, P. M., Boutron, I., Hoffmann, T. C., Mulrow, C. D., \& Moher, D. (2021). The PRISMA 2020 statement: An updated guideline for reporting systematic reviews. $B M J, 372, \mathrm{n} 71$.

Rokkas, T., Portincasa, P., \& Koutroubakis, I. E. (2018). Fecal calprotectin in assessing inflammatory bowel disease endoscopic activity: A diagnostic accuracy meta-analysis. Journal of Gastrointestinal and Liver Diseases, 27(3), 299-306.

Saluja, R., Cheng, S., Delos Santos, K. A., \& Chan, K. K. W. (2019). Estimating hazard ratios from published Kaplan-Meier survival curves: A methods validation study. Research Synthesis Methods, 10(3), 465-475.

Sanchez-Munoz, F., Dominguez-Lopez, A., \& YamamotoFurusho, J. K. (2008). Role of cytokines in inflammatory bowel disease. World Journal of Gastroenterology, 14(27), 4280-4288.

Sartor, R. B., \& Wu, G. D. (2017). Roles for intestinal bacteria, viruses, and fungi in pathogenesis of inflammatory bowel diseases and therapeutic approaches. Gastroenterology, 152(2), 327-339.

Sharon, G., Cruz, N. J., Kang, D. W., Gandal, M. J., Wang, B., Kim, Y. M., \& Mazmanian, S. K. (2019). Human gut microbiota from autism spectrum disorder promote behavioral symptoms in mice. Cell, 177(6), 1600-1618.

Shaw, K. A., Maenner, M. J., \& Baio, J. (2020). Early identification of autism spectrum disorder among children aged 4 years-Early autism and developmental disabilities monitoring network, six sites, United States, 2016. Morbidity and mortality weekly report. Surveillance Summaries, 69(3), 1.

Solmi, M., Firth, J., Miola, A., Fornaro, M., Frison, E., FusarPoli, P., \& Correll, C. U. (2020). Disparities in cancer screening in people with mental illness across the world versus the general population: Prevalence and comparative meta-analysis including 4 717839 people. The Lancet, 7(1), 52-63.

Somekh, J., Peleg, M., Eran, A., Koren, I., Feiglin, A., Demishtein, A., \& Elazar, Z. (2016). A model-driven methodology for exploring complex disease comorbidities applied to autism spectrum disorder and inflammatory bowel disease. Journal of Biomedical Informatics, 63, 366-378.

Sterne, J. A., Sutton, A. J., Ioannidis, J. P., Terrin, N., Jones, D. R., Lau, J., \& Higgins, J. P. (2011). Recommendations for examining and interpreting funnel plot asymmetry in meta-analyses of randomised controlled trials. $B M J, 343, \mathrm{~d} 4002$.

van't Hof, M., Tisseur, C., van Berckelear-Onnes, I., van Nieuwenhuyzen, A., Daniels, A. M., Deen, M., \& Ester, W. A. (2021). Age at autism spectrum disorder diagnosis: A systematic review and meta-analysis from 2012 to 2019. Autism: The International Journal of Research and Practice, 25(4), 862-873.

Vavricka, S. R., Spigaglia, S. M., Rogler, G., Pittet, V., Michetti, P., Felley, C., \& Straumann, A. (2012). Systematic evaluation of risk factors for diagnostic delay in inflammatory bowel disease. Inflammatory Bowel Diseases, 18(3), 496-505.

Walker, S. J., Fortunato, J., Gonzalez, L. G., \& Krigsman, A. (2013). Identification of unique gene expression profile in children with regressive autism spectrum disorder (ASD) and ileocolitis. PLoS One, 8(3), e58058.

Wells, G. A., Shea, B., Connell, D. O., Peterson, J., Welch, V., Losos, M., \& Tugwell, P. (2021) The Newcastle-Ottawa scale (NOS) for assessing the quality of nonrandomised studies in meta-analyses. Ottawa: Ottawa Hospital Research Institute. Retrieved from: http:// www.ohri.ca/programs/clinical_epidemiology/oxford.asp.

Williams, B. L., Hornig, M., Buie, T., Bauman, M. L., Cho Paik, M., Wick, I., \& Lipkin, W. I. (2011). Impaired carbohydrate digestion and transport and mucosal dysbiosis in the intestines of children with autism and gastrointestinal disturbances. PLoS One, 6(9), e24585.

Zaharie, R., Tantau, A., Zaharie, F., Tantau, M., Gheorghe, L., Gheorghe, C., \& Dobru, D. (2016). Diagnostic delay in Romanian patients with inflammatory bowel disease: Risk factors and impact on the disease course and need for surgery. Journal of Crohn's \& Colitis, 10(3), 306-314.

\section{SUPPORTING INFORMATION}

Additional supporting information may be found in the online version of the article at the publisher's website.

How to cite this article: Kim, J. Y., Choi, M. J., Ha, S., Hwang, J., Koyanagi, A., Dragioti, E., Radua, J., Smith, L., Jacob, L., de Pablo, G. S., Lee, S. W., Yon, D. K., Thompson, T., Cortese, S., Lollo, G., Liang, C.-S., Chu, C.-S., Fusar-Poli, P., Cheon, K.-A., ... Solmi, M. (2021). Association between autism spectrum disorder and inflammatory bowel disease: A systematic review and meta-analysis. Autism Research, 1-13. https:// doi.org/10.1002/aur.2656 